\title{
Diseño y construcción de un ecosistema digital: estrategias para articular la información y la formación policial
}

\section{Design and construction of a digital ecosystem: strategies to articulate police information and training \\ Desenho e construção de um ecossistema digital: estratégias para articular informação e treinamento policial}

\author{
Oscar Andrés Cárdenas Peña* \\ https://orcid.org/0000-0002-5883-7300 Policía Nacional de Colombia, Bogotá, Colombia
}

- Fecha de recepción: 2021-02-25

- Fecha concepto de evaluación: 2021-05-21

- Fecha de aprobación: 2021-05-23

https://doi.org/10.22335/rlct.v13i3.1417

\begin{abstract}
Para citar este artículo/To reference this article/Para citar este artigo: Cárdenas, O. A. (2021). Diseño y construcción de un ecosistema digital: estrategias para articular la información y la formación policial. Revista Logos Ciencia \& Tecnología, 13(3), 71-58.

https://doi.org/10.22335/rlct.v13i3.1417
\end{abstract}

\section{RESUMEN}

El presente artículo expone los resultados del proyecto orientado a la construcción de un ecosistema digital, para la consolidación y unificación de la información sobre los procesos y servicios formativos de la Dirección Nacional de Escuelas (DINAE) de la Policía Nacional de Colombia. El objetivo fue definir la estructura pertinente para la elaboración y desarrollo de este entorno, a partir de un diagnóstico con estudiantes, profesores, directivos y padres de familia como potenciales usuarios de los medios tecnológicos de las escuelas. En los resultados se encontró que el diseño del ecosistema renovó la gestión tecnológica de la institución, así como los procesos operacionales y la administración del servicio al cliente interno. De igual manera, se logró la convergencia de ocho componentes alojados en el portal educativo, permitiendo una mejor integración y articulación dentro del apoyo académico ofrecido. A partir de ahí, las tecnologías surgen como un soporte para las buenas prácticas de información, participación, colaboración, búsqueda y construcción de canales de comunicación más eficaces.

Palabras clave: Diseño tecnológico, ecosistema digital, información, formación policial 


\begin{abstract}
This article presents the results of the project aimed at the construction of a digital ecosystem, for the consolidation and unification of information on the training processes and services of the National Directorate of Schools (DINAE) of the National Police of Colombia. The objective was to define the pertinent structure for the elaboration and development of this environment, based on a diagnosis of the students, teachers, directives and parents, as potential users of the technological means of the schools. The results found that the ecosystem design renewed the institution's technological management, as well as the operational processes, and internal customer service administration. Similarly, it achieved the convergence of eight components housed in the educational portal, allowing a better integration and articulation within the academic support offered. From there, technologies emerge as a support for good practices in information, participation, collaboration, search, and construction of more effective communication channels.
\end{abstract}

Keywords: Technological design, digital ecosystem, information, training police

\title{
RESUMO
}

Este artigo apresenta os resultados de um projeto voltado para a construção de um ecossistema digital, para a consolidação e unificação da informação sobre os processos e serviços de treinamento da Direção Nacional de Escolas (DINAE) da Polícia Nacional da Colômbia. O objetivo foi definir a estrutura pertinente para a elaboração e desenvolvimento deste ambiente, a partir de um diagnóstico com estudantes, professores, diretores e pais de família, considerados usuários potenciais dos meios tecnológicos das escolas. Os resultados mostraram que o desenho do ecossistema renovou a gestão tecnológica, os processos operacionais e a administração do atendimento ao cliente interno da instituição. Da mesma forma, alcançou-se a convergência de oito componentes alojados no portal educacional, permitindo uma melhor integração e articulação dentro do suporte acadêmico oferecido. A partir daí, as tecnologias surgem como suporte de boas práticas de informação, participação, colaboração, busca e construção de canais de comunicação mais eficazes.

Palavras-chave: Design tecnológico, ecossistema digital, informação, treinamento policial.

Los ecosistemas digitales han definido un nuevo marco social, económico y cultural gracias al impulso tecnológico y a la masificación de internet, que genera la interacción permanente entre los usuarios. Esta situación está mediada por la infraestructura, los servicios y las aplicaciones, como elementos centrales para la conformación de estos entornos. Dichos ecosistemas también se encuentran presentes en los entornos educativos, respaldados por una política de gobierno digital, que parte de un proceso orientado a promover las tecnologías de la información y comunicación (en adelante TIC) en la formación de ciudadanos y en el uso que hacen de estos medios (ITU, 2017).

En el campo empresarial, los ecosistemas digitales integran el funcionamiento de algunos métodos y técnicas para el fortalecimiento del proceso de atención y servicio de las compañías. Autores como López (2005), Pineda y Orozco (2017) sostienen que estos entornos constituyen un sistema dinámico y complejo, donde las personas se ven condicionadas por las diferentes formas de mediación que impactan su pensamiento y su modo de relacionarse con los demás.

En efecto, las instituciones sociales y educativas de Colombia se han visto convocadas para fortalecer el manejo de la información y su centralización. Desde su creación, el Ministerio de Tecnologías de la Información y las Comunicaciones (MinTIC) ha sido el encargado de abanderar programas dirigidos al uso responsable y pedagógico de las TIC, tales como: Computadores para educar y En TIC confío, además de integrar los puntos Vive Digital a su propuesta de demanda tecnológica de estos proyectos (MinTIC, 2018a). Frente a esta situación, especialmente en el marco de la emergencia causada por el covid-19, se han debido repensar las estrategias para garantizar una alfabetización digital coherente y oportuna. 
Actualmente, la incidencia de las políticas adelantadas por MinTIC también tiene que ver con el tratamiento de datos, por ejemplo, frente el manejo de la pandemia y la atención ciudadana por medios electrónicos. Sumado a eso, se puede considerar el fortalecimiento de escenarios para brindar información al sector académico, así como la reactivación económica pensada desde las categorías de emprendedores y el sector industrial en el país (MinTIC, 2018a).

Para el caso de la Dirección Nacional de Escuelas de la Policía Nacional (en adelante DINAE), el diseño y construcción de un ecosistema digital representa una estrategia que integra los servicios sobre procesos de formación. Con el propósito de atender las necesidades de la población que conforma la comunidad académica, este escenario estructura, de manera lógica y sistemática, los cuatro componentes que conforman un ecosistema digital: usuarios, infraestructura, servicios y aplicaciones, añadiendo a ello la gestión académica de la comunidad.

De esta manera, el diseño, elaboración y puesta en marcha del ecosistema digital contempla y mantiene el valor de la sinergia entre tecnología, comunicación y pedagogía, aspectos que deben confluir como espacios de interacción y apertura a la innovación (Policía Nacional de Colombia, 2013). A pesar de los avances educativos al interior de la Policía Nacional, tales como la creación de bases de datos sólidas para la interacción de usuarios, al iniciar este proyecto la institución no contaba con una propuesta TIC integradora que involucra los componentes que orientan la creación de proyectos (MinTIC, 2019).

La implementación del ecosistema digital se basó en un producto para la optimización de los procesos académicos y de administración educativa para la organización. Este medio brinda información sobre la toma de decisiones para la gestión de herramientas académicas. Por este motivo, el proyecto se ha ocupado de diseñar una propuesta articulada con la misión DINAE (Policía Nacional de Colombia, 2019), a saber:

Direccionar la formación del talento humano de la Policía Nacional, a través del sistema educativo policial, en cumplimiento de las funciones de docencia, investigación y proyección social, con el fin de contribuir a la satisfacción de las necesidades de convivencia.

El ecosistema digital surge, bajo la perspectiva de Pineda y Orozco (2017), como un insumo para la consolidación de las metas fijadas para responder a las exigencias tecnológicas, a través de un acompañamiento activo a la institución en la prestación del servicio. Por consiguiente, en el marco de la apropiación de las TIC y de su contribución a los entornos sociales y educativos, se planteó la siguiente pregunta que orientó la propuesta: ¿cómo diseñar un ecosistema digital para fortalecer los procesos formativos, articulando los servicios académicos de la DINAE de la Policía Nacional de Colombia?

A partir de esta pregunta se propuso, como objetivo general, la identificación de los aspectos constitutivos de un ecosistema digital, para fortalecer los procesos de formación articulados al servicio académico de la DINAE de la Policía Nacional de Colombia. En el presente artículo se realiza un balance bibliográfico, donde se describen diferentes perspectivas y resultados de investigaciones sobre ecosistemas digitales. Asimismo, se evidencian los aportes desde distintos contextos institucionales y educativos al tema de interés.

Posteriormente, se expone la ruta de trabajo utilizada para el levantamiento de información (fase diagnóstica), que sirvió como insumo para el diseño, elaboración y puesta en marcha del ecosistema digital. En el apartado final del documento se exponen los resultados de la fase diagnóstica, con los cuales se dio lugar al diseño del ecosistema digital de la DINAE, así como las proyecciones derivadas del proceso realizado.

\section{Antecedentes}

El diseño y puesta en marcha de los ecosistemas digitales ha sido un tema abordado en distintos escenarios, con propuestas innovadoras frente a problemáticas en contextos diversos. Dentro de este apartado se presenta el resultado del rastreo bibliográfico realizado, con el cual se analizaron las tendencias sobre estructuración, organización y aportes de estos ecosistemas en las instituciones en que fueron implementados, especialmente las de naturaleza educativa.

Los resultados de esta búsqueda inician con investigaciones que abordan el tema de ecosistemas digitales en escenarios sociales amplios, hasta llegar a estudios que, específicamente, se centran en entornos educativos. A partir de ahí, se logran identificar características comunes de estos ecosistemas, sus componentes y aportes en diferentes organizaciones, teniendo en consideración las características híbridas de la DINAE como entidad estatal, institución de la policía y escenario educativo, simultáneamente. 
La investigación realizada por la ITU (2017) planteó como objetivo el análisis de la experiencia de Paraguay desde un entorno tecnológico, respecto al uso y aplicabilidad de las TIC en diversas áreas, considerando la perspectiva social y económica del país. Bajo una metodología de naturaleza cualitativa y un estudio de caso se realizó un diagnóstico, que más tarde fue replicado en distintos países de América Latina, en tres momentos de análisis: inicialmente, se compiló información para determinar las regulaciones normativas, técnicas y estadísticas; luego se realizaron diferentes recorridos para recolectar información sobre los cuatro componentes que conforman el ecosistema digital y, finalmente, se presentó la organización documental, además del análisis y las recomendaciones. Entre los resultados obtenidos, se reconoce la necesidad de desarrollar indicadores de seguimiento sobre la evolución de la masificación de las tecnologías, así como la importancia de fortalecer los mecanismos de protección al consumidor y de interacción entre las empresas prestadoras de servicios.

Por otro lado, Carrera et al. (2013) presentan los resultados de una investigación con enfoque cualitativo, donde se da cuenta de la importancia de los medios de comunicación y la relación de los usuarios a partir de información estructurada. En este orden, se valora la participación de las personas quienes, al ser prosumidores de los diferentes ecosistemas digitales a los que se enfrentan, caracterizados por configurar una nueva lógica narrativa no lineal y universal. En las conclusiones, los autores definen el storytelling transmedia como una forma narrativa que integra al internet con los medios de comunicación tradicionales. También señalan una perspectiva desde la cual la dimensión pública del uso del internet supera la conversación mediática patrimonial y exclusiva de pocas personas. El objetivo es pensar un sistema que permita la gestión del flujo comunicativo, donde el consumo, la creación, la administración de la información y la disposición de la sociedad sean acciones indispensables para desenvolverse en los sistemas comunicativos actuales.

Por otra parte, el Centro Nacional de Consultoría de Colombia (Rey, 2013) realizó una encuesta de cultura digital que da cuenta de las recientes transformaciones de un ecosistema mediático a otro digital, donde las nuevas tecnologías, su uso y las producciones digitales han sido un elemento fundamental. En los últimos años, se reconoce que los avances tecnológicos han tenido un alto crecimiento en niveles cualitativos y cuantitativos. En términos cuantitativos, se observa el aumento de las redes alámbricas, inalámbricas y satelitales, así como de los equipamientos tecnológicos, en particular de los dispositivos móviles, y la relación de uso con ellos por personas y hogares. A nivel cualitativo, se muestra que están apareciendo nuevas prácticas culturales de interacción, apropiación y uso de servicios mediados por tecnologías en diversos ámbitos. En el ámbito de la educación, los cambios han sido más profundos, porque los medios y los contenidos, ahora digitales, han tomado el lugar de lo instrumental y lo ilustrativo. De ser un elemento que pueda complementar o simplemente dar soporte a una enseñanza, los ecosistemas digitales se han transformado en el espacio propicio para generar la interactividad, así como un aprendizaje más crítico, provechoso y participativo. En virtud de ello, el uso cotidiano de las nuevas tecnologías ha configurado nuevas formas de acceder a la información, y su impacto atiende a las necesidades de los usuarios gracias a la conectividad y a las mayores posibilidades de navegación. El estudio reconoce que, aunque aún existen dificultades en el país en relación con la conectividad y el desarrollo de competencias digitales ciudadanas, sigue una tendencia creciente en el uso de los ecosistemas digitales. Su enfoque se centra en el servicio a los ciudadanos por parte de distintas instituciones estatales, policiales y militares, lo que permitirá el fortalecimiento de una gobernanza digital y la interacción de la ciudadanía con las agencias del Estado, mediante la implementación de mecanismos de participación en la red (Rey, 2013).

De otro lado, Cáceres, Brändle y Ruiz (2016) consideran que los entornos o ecosistemas digitales corresponden, actualmente, a la normalización del uso de las TIC en la vida cotidiana. El desarrollo tecnológico, según afirman, ha permitido formas habituales de socializar, expresando, consecuentemente, la manera "natural" y contemporánea de "estar en" el mundo. Los autores sostienen que en esta sociedad todos los ciudadanos están hiperconectados ininterrumpidamente, hecho que da lugar a pensar en una sociabilidad digital. Con la inserción de las nuevas tecnologías en los diversos escenarios y procesos, estos cambios han afectado las relaciones establecidas entre los sujetos participantes. El impacto más trascendente es la reconfiguración sobre las diferentes formas de comunicación, los espacios de interacción y las dinámicas de relacionamiento entre los sujetos. Siendo así, el plano social y cultural se ha transformado desde maneras de vincularse o afiliarse con otros.

En cuanto a los estudios documentales, Islas (2017) realizó un análisis de la literatura existente sobre los ecosistemas digitales. El método de revisión aplicada incluyó 
dos fases: heurística y hermenéutica. La primera etapa consideró los estudios científicos en español, publicados en revistas indexadas entre los años 2009 y 2017. En la segunda fase, la metodología consistió en el análisis, clasificación e interpretación respecto a qué son los ecosistemas digitales, su aprendizaje y la relación con la sociedad del conocimiento. La conclusión es que la literatura expresa el cómo debe ser un ecosistema digital, pero se constata la carencia de suficientes datos empíricos que ayuden a comprender y evidenciar sus aportes más significativos.

En el estudio realizado por Motz y Rodés (2013) se planteó una revisión y análisis de la literatura pertinente. El interés de esta investigación era establecer la diferencia entre un ecosistema digital de aprendizaje (EDA) de aqueIlo que se define como Entorno Virtual de Aprendizaje (EVA). Los autores reconocen que, si bien semánticamente son cercanos, expresan realidades distintas, que reflejan una evolución específica en la manera de conceptualizar un ecosistema digital, las condiciones que presentan y los factores que influyen en su construcción. En un EVA, como por ejemplo Moodle, los contenidos solo son accesibles mientras el entorno está dispuesto para un proceso de enseñanza y aprendizaje. Además, su exportación es compleja, o puede no estar contemplada, por ser un sistema cerrado. Este estudio constató que el uso por parte de los profesores en un EVA se asemeja más a un repositorio de materiales o recursos, donde la administración y la gestión de contenidos es predominante. Contrario a esto, en un EDA se configuran otras condiciones e intervienen factores externos e internos heterogéneos que lo convierten en la última generación de lo que se comprende como ecosistema digital; es decir, posee características propias como adaptabilidad, interconexión y producción de servicios, compartiendo abiertamente los recursos y promoviendo la reflexión y participación de los usuarios o participantes. Es importante considerar que el EDA tiene bases teóricas y pedagógicas del conectivismo y del socio-constructivismo, como factores que aportan al fortalecimiento de las prácticas institucionales.

Desde las posibles relaciones entre educación y ecosistema digital, Díaz (2016) resalta la importancia de la interacción o interconexión como un elemento fundamental, que debe favorecerse entre participantes o usuarios. Esto dependerá de la eficacia de la experiencia educativa que se proponga y las posibilidades que permita; además, de quienes participen o hagan uso de sus contenidos. La autora señala como factor determinante para este éxito la planificación educativa institucional, dado que un ecosistema digital responde a un contexto particular y a perfiles de usuario específicos.

Precisamente, la interconectividad como elemento fundamental en el uso de las tecnologías es el aspecto que ha favorecido la creación y evolución de los ecosistemas digitales desde la década de los noventa. Asimismo, es relevante señalar que las instituciones deben ser capaces tanto de ofrecerlas como de innovarse permanentemente, a fin de constituir respuestas adaptativas y dinámicas a las situaciones o necesidades particulares.

Entre los aportes que ofrece un ecosistema digital a un determinado proceso de aprendizaje diverso está, en primer lugar, la posibilidad de brindar un modelamiento del escenario en el que se aprende, utilizando un entorno virtual y las herramientas tecnológicas necesarias para dar soporte al proceso mismo de enseñanza-aprendizaje. En segundo lugar, la constitución de un ambiente propicio para la adquisición de habilidades y competencias (genéricas y transversales) digitales, que favorecen el aprendizaje constante.

De igual manera, los ecosistemas digitales permiten la generación de rutas de aprendizaje contextualizadas y personalizadas. Es así como a partir de las necesidades y/o situaciones de contexto particular se generan ofertas, recursos y servicios, según el perfil de sus participantes o de los usuarios. Finalmente, se destaca la contribución a una educación más activa y participativa, constructiva y colaborativa. Estos aspectos educativos, tanto en la manera de enseñar como de aprender, se ven profundamente afectados con la propuesta de un ecosistema digital (ITU, 2017).

Por su parte, Barrios y Chaves (2016) contemplan el nivel de exigencia de los docentes al incluir el uso de las TIC en el proceso de formación, como apoyo a las clases presenciales y al modelo de educación en el que se insertan. Con el fin de dar explicación a esta situación, se desarrolló una investigación mixta, bajo un enfoque experimental, con estudiantes y docentes de Comunicación Social y Periodismo. El propósito fue reconocer el papel de las nuevas tecnologías en el aula y la idea que tienen las personas que participan en el proceso formativo de cara al uso de estas herramientas. En este estudio se concluyó que, en el modelo presencial, lo tecnológico se convierte en una herramienta que complementa al proceso de enseñanza-aprendizaje. El modelo virtual o de educación a distancia, por su parte, se plantea como un paradigma de la didáctica tecnológica. 
La propuesta de Islas (2019) es un abordaje sistémico para el fenómeno que originan los ecosistemas digitales, desde las acciones de los estudiantes respecto a su aprendizaje y la interacción que se da entre ellos, los docentes, los contenidos y los medios. Esto permite una aproximación de observación y explicación para la conexión entre los individuos y su contexto, resaltando la retroalimentación que se genera a través de diferentes canales de mediación que evolucionan dentro de un ambiente. La propuesta se sustenta en una serie de elementos observables, como son las habilidades tecnológicas, las 4C, las competencias transversales y los factores sociales que operan sobre quienes participan.

En contraste, Ospina y Galvis (2017) presentan un ecosistema digital para la educación, a partir de diferentes referentes teóricos. Estos autores articulan siete componentes, cuya función es representar el diseño propuesto desde un sistema dinámico que se ve influenciado por la interacción de cada componente. El modelo de ecosistema se está probando en un entorno $\mathrm{EVA}^{1}$ y en la primera etapa se enfoca en la exploración de la dinámica del ecosistema digital, mediante la prueba de conferencias virtuales y la interacción de los tecnólogos en el ambiente de aprendizaje.

Desde esta perspectiva, Morales (2018) establece nuevos ambientes de formación, basados en plataformas virtuales o cursos en línea masivos y abiertos, y compara esta nueva alternativa de prácticas pedagógicas con los modelos que han permanecido durante las últimas décadas. Igualmente, analiza la adaptabilidad de estos modelos como nuevos ecosistemas digitales, tanto en el ámbito de educación superior universitaria como en usuarios no universitarios, permitiéndoles otras posibilidades que les facilitan el aprendizaje y la comunicación.

Dentro de su propuesta, Ramírez y Chacón (2018) se ocuparon de revisar la deficiente calidad de la educación en Colombia, especialmente en las áreas rurales, debido a las marcadas diferencias sociales, económicas y educativas. Los autores proponen la conceptualización de los ecosistemas digitales como una herramienta que permitiría asegurar la cobertura y calidad en estas regiones y los múltiples retos que esto presenta, pero apostando a su implementación en las escuelas y al impacto que supone esta práctica.

1 En el enlace http://e-ducate.co se encuentra la propuesta diseñada por Ospina y Galvis (2017).
Finalmente, Ortiz et al. (2017) realizan un análisis sobre el uso de un ecosistema digital de apoyo a niños con dificultades de aprendizaje en lectura y matemáticas básicas, desde la perspectiva de que la tecnología está presente en casi todas las actividades del ser humano, porque aporta herramientas para realizar las tareas de la casa, el entretenimiento, el trabajo, la investigación, entre otras, y la educación no está excluida en esta importante apreciación. El estudio que realizan estos autores pretende acercar la tecnología a la comunidad a través de un modelo de ecosistema digital, a partir de la retroalimentación realizada por los mismos usuarios. El reto consistió en revisar la gran cantidad de recursos educativos disponibles y la necesidad de organizar, administrar y evaluar dichos recursos para su óptimo aprovechamiento.

Ante este panorama, el rastreo bibliográfico señala como principal aporte de los ecosistemas digitales la capacidad de favorecer procesos formativos en distintos contextos educativos. De igual manera, se considera su potencial para construir en escenarios significativos para el desarrollo y fortalecimiento de competencias digitales, donde se permita el uso de entornos digitales en procesos educativos, es decir, la habilitación de espacios de manejo y disposición de la información, donde la interacción sea predominante y constitutiva de los ecosistemas digitales.

\section{Marco teórico}

\section{Ecosistema digital}

Un ecosistema digital está asociado con procesos soportados en las TIC, y se caracteriza por basarse en sistemas adaptativos capaces de modificarse a partir de las relaciones entre los componentes del sistema: aplicaciones, usuarios, servicios e infraestructura (Magro et al., 2014; ITU, 2017). El concepto mismo implica la construcción de nuevos paradigmas mediáticos, capaces de materializar otras formas de relacionamiento, participación y estimulación democrática y participativa dentro de las estructuras digitales.

Diferentes autores (Aguado \& Navarro, 2013; López, 2005) sostienen que la metáfora del ecosistema digital resignifica la sociedad existente, no solo como una comunidad de seres vivos que comparten un medio. Más allá de esto, representa una colectividad interconectada que utiliza las herramientas tecnológicas con distintos fines. Igualmente, dichos autores analizan la manera en que estos recursos desarrollan o conllevan a nuevos procesos 
y comportamientos económicos, educativos y culturales, propios de la sociedad del conocimiento.

Particularmente, el Gobierno nacional de Colombia, en cabeza del MinTIC, ha liderado un conjunto de políticas y proyectos encaminados a concebir los ecosistemas digitales como espacios para la formación en democracia digital, y para la promoción y el fortalecimiento de la seguridad de la información. También se considera la construcción de redes de telecomunicaciones que promuevan el comercio, la educación, la inclusión social y la participación digital (MinTIC, 2018b).

A partir de los planteamientos de MinTIC que orientaron el diseño y elaboración del ecosistema digital para la DINAE de la Policía Nacional, se describen cuatro componentes esenciales y constitutivos de los ecosistemas digitales, que se describen a continuación.

\section{Componentes del ecosistema digital}

La Fundación Telefónica (2016) ha sido la encargada de analizar a profundidad la relación que existe entre los cuatro componentes del ecosistema digital, desde su origen, con las problemáticas que reviste su regulación como una política TIC. En la figura 1 se presentan las principales características de estos elementos: infraestructura, servicio, aplicaciones y usuarios.

Figura 1

Cuatro componentes del ecosistema digital

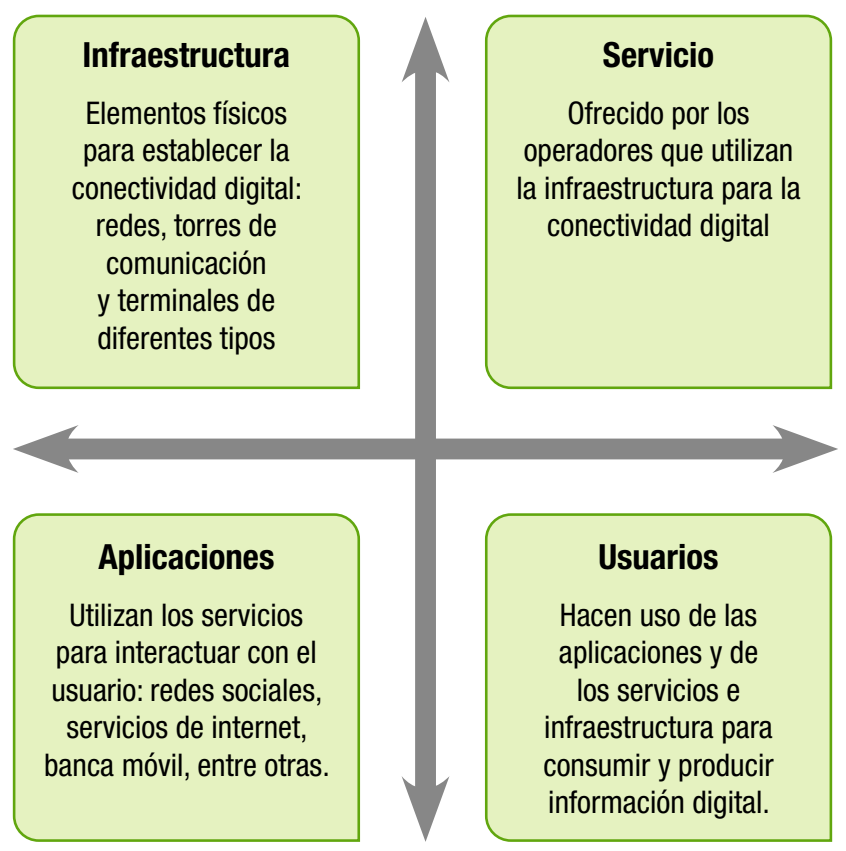

Si bien los cuatro componentes del ecosistema digital funcionan de manera integral, dada su interacción permanente, para el caso de la DINAE de la Policía Nacional se hizo énfasis en el análisis del componente de servicios, es decir, de la oferta, al igual que en el modo de divulgación y apoyo de esta para los usuarios finales, dado el carácter y necesidades del contexto en el que se implementa el ecosistema. En los siguientes apartados se explican los aspectos más significativos de este componente de servicios.

Componente de servicio en el marco institucional Para este proyecto se hizo la elección, únicamente, del componente de servicio. Esta elección obedece a una delimitación específica, según la necesidad de la institución, orientada a determinar aspectos tales como: la población, la cobertura, la difusión y las fuentes de información de la oferta académica constitutiva del ecosistema digital. Dentro de la figura 2 se especifican las acciones y los métodos relacionados con estos aspectos.

Figura 2

Aspectos del componente de servicios

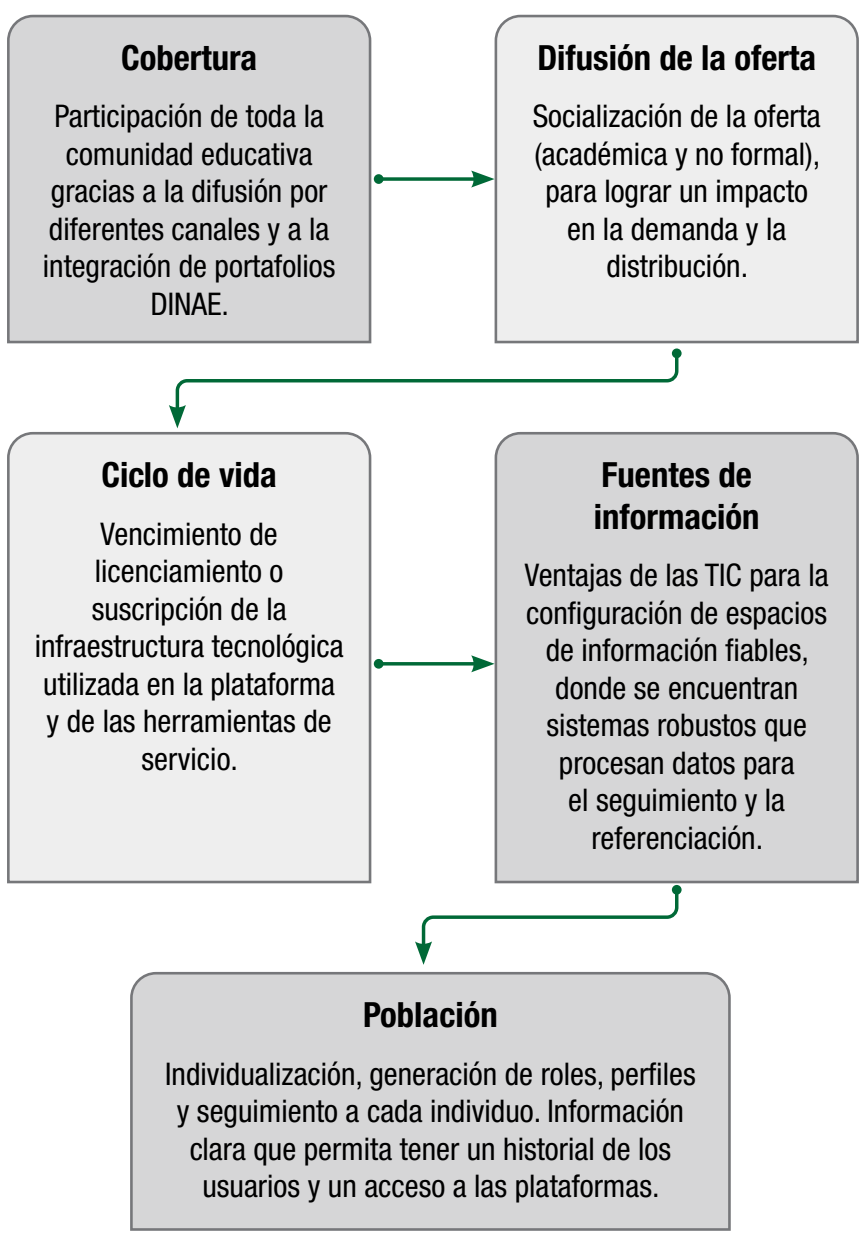


Con lo anterior, se evidencia que el componente de servicios constituye un insumo importante para el dimensionamiento de los requerimientos de hardware, software y necesidades de capacitación. Gracias a estas características se pueden optimizar los perfiles y las competencias de servicio de los policías que se encuentran en el área académica, así como de los recursos que contribuyen significativamente en la prestación de su ejercicio.

\section{Marco metodológico}

La propuesta se enmarca en una metodología de carácter mixto, por el uso de instrumentos de naturaleza cualitativa y cuantitativa con alcance descriptivo-reflexivo. Este tipo de investigación se caracteriza por explorar los fenómenos desde la perspectiva de sus participantes y su relación con el contexto (Hernández, Fernández \& Baptista, 2015). Además, se orienta a reconocer y analizar las experiencias desde el punto de vista de los sujetos que hacen parte del escenario de estudio. Este enfoque permitió observar los resultados de la implementación del ecosistema digital con el cliente interno y académico de la DINAE, en el componente de servicios digitales.

En este estudio mixto, algunos de los instrumentos miden opiniones, perspectivas y acciones desde un análisis cuantitativo (encuestas); otros, bajo una mirada cualitativa (entrevista). No obstante, el interés recae en la búsqueda de datos descriptivos y reflexivos sobre la incorporación y operacionalización del ecosistema digital, a partir de la posibilidad de explicar y analizar diferentes situaciones de los servicios académicos que presta la DINAE (Hernández et al., 2015).

La ruta metodológica consistió en la toma de información sobre las necesidades específicas de los usuarios, en relación con el uso y articulación de los diferentes productos y servicios, mediados por TIC, ofrecidos en la DINAE de la Policía Nacional. Este ejercicio se planteó para identificar los elementos más importantes a tener en cuenta en el diseño, elaboración y propuesta del ecosistema digital, en consideración de sus experiencias y opiniones.

En cuanto a las técnicas e instrumentos para la recolección de la información, se utilizó una encuesta llamada Planes TIC, la cual se implementó por medio de un cuestionario de Google Drive para todas las escuelas de formación de la Policía Nacional, el 7 de junio de 2018. A cada usuario se le informó del proceso de investigación y de sus alcances por medio del correo institucional con dominio@correo.policia.edu.co; luego se envió el cuestionario y se recolectó la información para su posterior análisis.

En la tabla 1 se presenta un ensamble con los principales elementos metodológicos que orientaron esta propuesta: enfoque, diseño, técnica e instrumentos.

Tabla 1

Ensamble de componentes metodológicos

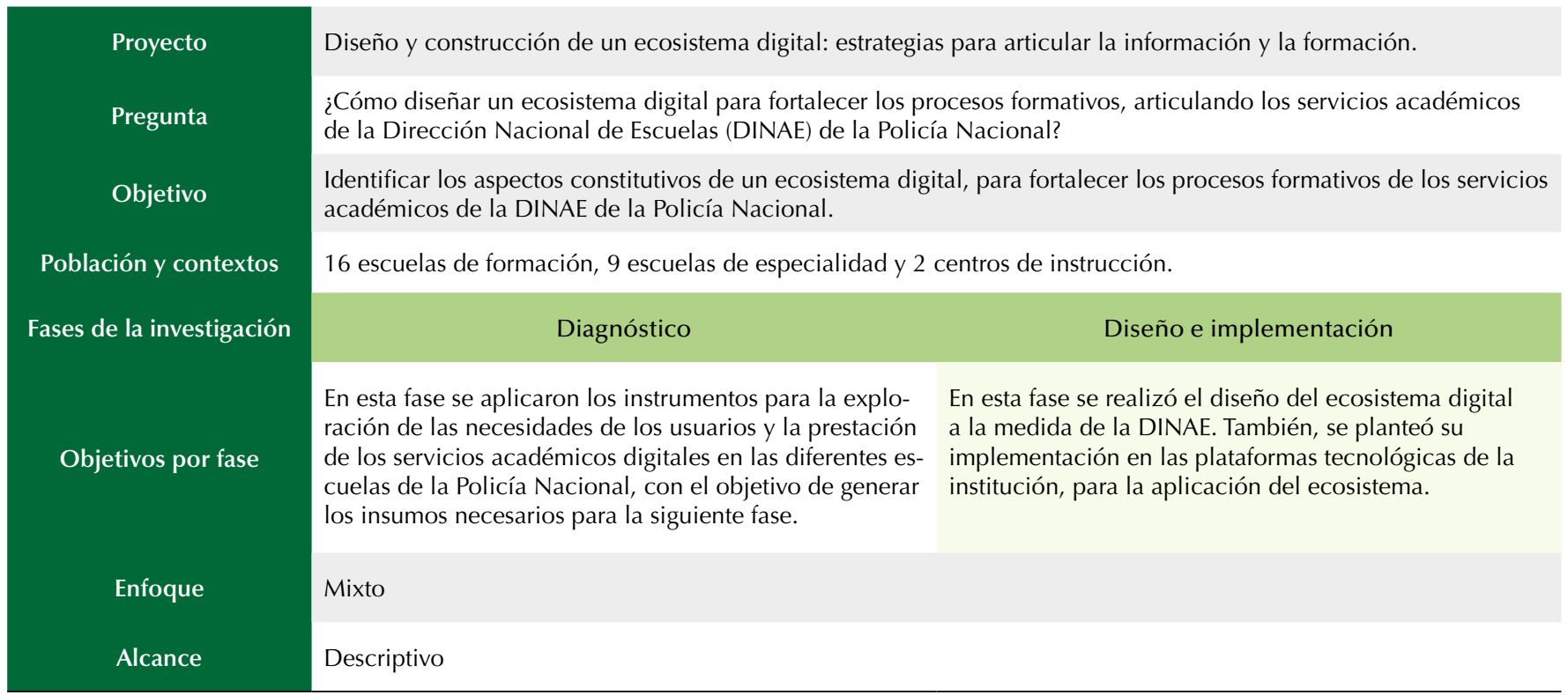

Nota: adaptado de Jiménez (2020). 
Con el propósito de obtener información relevante de los diferentes usuarios, la población de estudio estuvo conformada por estudiantes, docentes, directivos académicos y padres de familia. En cuanto a la muestra, se realizó una elección no probabilística por conveniencia, porque su selección obedece a los intereses y propósitos de la investigación; además, por la disponibilidad y por la cercanía con el investigador (Hernández et al., 2010). Se contó con la participación de 16 escuelas de formación policial pertenecientes a la DINAE, 9 de especialidades y 2 centros de instrucción de la Policía Nacional. La siguiente categorización evidencia el trabajo desarrollado con el personal de:

- Dieciséis escuelas de formación, encargadas de los procesos de formación y capacitación policial a nivel nacional (Bogotá, Sibaté, Espinal, Corozal, Villavicencio, Barranquilla, Manizales, Tuluá, Medellín, Fusagasugá, Vélez y Santa Rosa de Viterbo).

- Nueve escuelas de especialidades como: apoyo a los procedimientos de servicio de policía para fortalecer temas de seguridad vial, investigación criminal, inteligencia, telemática, protección a dignatarios, prevención y reacción al secuestro y extorsión, y formación de pilotos desplegados en Mariquita (Tolima).

- Dos centros de instrucción, donde se forman policías que apoyan comunidades con alguna particularidad étnica, como el centro de instrucción wayú en La Guajira y en el Chocó.

\section{Resultados}

Atendiendo a la ruta metodológica propuesta para el proyecto, en este apartado se presentan los resultados obtenidos en el diagnóstico, como un insumo para el diseño y construcción del ecosistema digital para la DINAE de la Policía Nacional. El objetivo general permitió no solo la identificación de aspectos constitutivos de un ecosistema digital para el fortalecimiento de procesos formativos de la DINAE, sino un desarrollo óptimo de procesos en la consolidación de espacios y contenidos virtuales que favorecieron el componente de servicio.

Concretamente, uno de los principales alcances de la propuesta tiene que ver con la convergencia y congregación de las capacidades desarrolladas en los procesos educativos de la comunidad académica de la Policía Nacional. En este orden de ideas, se desarrolló un sistema que integró el Sistema Nacional de Bibliotecas con bases de datos especializadas, importantes para la investigación, la movilización de información y el acercamiento al material bibliográfico, físico y digital. Además, la Red Nacional Académica de Tecnología Avanzada (RENATA) ofrece la conexión a la red nacional de investigación y educación, lo cual visibiliza el trabajo académico.

Sumado a lo anterior está el Repositorio Institucional, elemento constitutivo de identidad, dado que da cuenta de los avances investigativos cuya propiedad intelectual está afiliada a la Policía Nacional y Seguridad de la información. Esto posibilita el acceso a las políticas de información por parte de miembros de la organización. De este modo, el ecosistema digital habilitó un primer acercamiento a la problemática y representó una pauta de inicio para la divulgación de servicios digitales e infraestructuras modernas, a través de la creación de una sola interfaz institucional.

Como en efecto se revela la optimización de recursos a partir de la centralización de la información, varias entidades asociadas a la Policía generaron una red de consulta que garantizó una mejor inversión y el acceso a otras escuelas que no participaban allí. La optimización de recursos implica la interacción e interconexión entre escuelas para generar una economía en escala y gestionar de mejor forma los recursos, lo cual permite que los encargados del área tecnológica puedan asumir un seguimiento detallado de los alcances de la inversión y, por ende, evaluar el uso para la toma de decisiones.

Por último, la socialización de este tipo de aprendizajes e investigaciones institucionales favorecieron las capacidades de los policías, porque generan herramientas y oportunidades de mejora al servicio de la institución. Es sumamente significativo que estos instrumentos hayan sido desarrollados por personas pertenecientes a la comunidad académica de la Policía Nacional, pues esto garantiza que exista una capacidad instalada en el cuerpo de la institución, así como la flexibilidad y actualización del inventario único de servicios.

\section{Fase I. Diagnóstico}

En esta fase se realizó la encuesta de Google Drive, en la que participaron 250 personas que hacen parte de los procesos educativos desarrollados en la Policía Nacional. La información se presenta discriminada a continuación (tabla 2). 
Tabla 2

Grupos poblacionales: fase 1

\begin{tabular}{|cc|}
\hline Grupo focal & $\begin{array}{c}\text { Número y porcentaje } \\
\text { de participantes }\end{array}$ \\
\hline Docentes & $40(16 \%)$ \\
\hline Estudiantes & $145(55 \%)$ \\
\hline Directivos & $20(8 \%)$ \\
\hline $\begin{array}{c}\text { Padres de familia (de estudiantes } \\
\text { menores de edad). }\end{array}$ & $50(21 \%)$ \\
\hline
\end{tabular}

El siguiente reporte (tabla 3) evidencia la calificación obtenida en los distintos aspectos indagados desde la encuesta sobre el uso de las TIC, por parte de la población que trabaja, asiste y está vinculada a la DINAE.

Tabla 3

Calificación de las dimensiones de apreciación TIC

\begin{tabular}{|c|c|}
\hline Área & Promedio \\
\hline Gestión y planificación & 2.33 \\
\hline TIC y desarrollo curricular & 2.5 \\
\hline $\begin{array}{c}\text { Desarrollo profesional } \\
\text { de los docentes }\end{array}$ & 3.0 \\
\hline Cultura digital & 3.67 \\
\hline Recursos e infraestructura TIC & 4.71 \\
\hline Institución escolar y comunidad & 4.6 \\
\hline
\end{tabular}

La escala de evaluación para cada uno de los indicadores se ubicó en un rango de 1 a 5, siendo 1 la calificación más baja y 5 la más alta. En este sentido, las calificaciones expuestas en la tabla 3 evidencian que la percepción cuantitativa de los participantes, frente a la manera en que se utilizan las tecnologías en la DINAE, oscila en un rango bajo y medio-bajo. Los aspectos que presentan más bajo puntaje son los de gestión y planificación del uso de TIC, seguidos por la incorporación de las TIC en los procesos de desarrollo curricular de la institución.

En cuanto al desarrollo profesional de los docentes y la cultura digital institucional, los participantes valoraron con una escala promedio los procesos que se llevan a cabo en la DINAE. Por otra parte, se otorga una calificación alta a los aspectos relacionados con los recursos e infraestructura TIC con los que se cuenta en la organización, así como a la incorporación de estas tecnologías en la institución educativa y en la comunidad.
En relación con los docentes, estos manifiestan tener claridad sobre los beneficios pedagógicos que aportan las TIC. Como profesionales, reconocen y comprenden el potencial didáctico de las tecnologías y los beneficios que ofrece el ecosistema digital de la DINAE. Asimismo, destacan el trabajo colaborativo que surge como factor esencial y motivacional para la inclusión de las TIC en la educación (Jiménez, 2015).

Los estudiantes identificaron el ecosistema digital como una herramienta complementaria que facilita la apropiación del conocimiento. En su mayoría, los estudiantes son nativos digitales, es decir, se caracterizan como miIlennials o centennials y son expertos en el uso de herramientas tecnológicas y redes sociales (Prensky, 2001). El estudiantado, entonces, desempeña un papel central en la cadena de aprendizaje, lo que genera retos y desafíos para los directivos y docentes. En opinión de Jiménez (2018), esto conlleva a la apropiación de este tipo de herramientas para el desarrollo del pensamiento crítico y creativo en el aula.

Es importante anotar que los directivos TIC tienen conocimiento del ecosistema digital liderado por la DINAE. Sus percepciones permiten identificar el compromiso como facilitadores del ejercicio con docentes, estudiantes y directivos, dado que desarrollan actividades técnicas en las plataformas propuestas. Esto demanda una actualización, supervisión y control constante frente al proceso de enseñanza y aprendizaje de cada miembro de la organización (MinTIC, 2019).

La apreciación sobre la incorporación de las tecnologías de la información no es lo suficientemente favorable en todas las dimensiones institucionales. Este aspecto se confirma con otros instrumentos que fueron aplicados en la fase de diagnóstico. Se exploró acerca del uso que hacen los diferentes tipos de usuarios del Sistema Nacional de Bibliotecas, por ser uno de los servicios que la DINAE ofrece como apoyo a los procesos académicos. Los resultados arrojados por la encuesta (tabla 4), evidencian el acceso limitado al Sistema Nacional de Bibliotecas por parte de la comunidad académica; a pesar de contar con una cantidad suficiente de suscripciones (figura 3), muchas veces este recurso no es empleado como fuente de información.

Por esta razón, se concluye que hacen falta estrategias de promoción sobre las potencialidades de este tipo de herramientas para los estudiantes. Su uso puede generar un impacto positivo frente a la búsqueda de información 
para el aprendizaje; de ahí la necesidad de divulgar la implementación de este recurso en las aulas, especialmente en relación con las dinámicas impuestas por la coyuntura del covid-19.

\section{Tabla 4}

Resultado de las sesiones en el rango 2018-04-01 y 2018-06-08 (ranking de usuarios)

\begin{tabular}{cccc}
\hline $\begin{array}{c}\text { Escuela de } \\
\text { formación }\end{array}$ & I.D. Usuario & Identificación & Sesiones \\
\hline ESREY & $1045^{* * * * *}$ & $1045^{* * * * *}$ & 56 \\
\hline ESAGU & $997^{* * * *}$ & $997^{* * * *}$ & 41 \\
\hline ESPOL & $10142^{* * * *}$ & $10142^{* * * *}$ & 40 \\
\hline ESGAC & $1014^{* * * *}$ & $1014^{* * * *}$ & 36 \\
\hline ESEVI & $1005^{* * * * *}$ & $1005^{* * * * *}$ & 27 \\
\hline ESTEL & $1100^{* * * * *}$ & $1100^{* * * * *}$ & 26 \\
\hline ESMEB & $1077^{* * * * *}$ & $1077^{* * * * *}$ & 25 \\
\hline ESAVI & $1065^{* * * * *}$ & $1065^{* * * * *}$ & 25 \\
\hline ESGON & $1052^{* * * * *}$ & $1052^{* * * * *}$ & 25 \\
\hline ESCER & $1052^{* * * * *}$ & $1052^{* * * * *}$ & 25 \\
\hline ESECU & $1055^{* * * * *}$ & $1055^{* * * * *}$ & 24 \\
\hline ESBOL & $1052^{* * * * *}$ & $1052^{* * * * *}$ & 23 \\
\hline ESCIC & $1007^{* * * * *}$ & $1007^{* * * * *}$ & 23 \\
\hline ESJIM & $1055^{* * * * *}$ & $1055^{* * * * *}$ & 23 \\
\hline ESREY & $1056^{* * * * *}$ & $1056^{* * * * *}$ & 23 \\
\hline
\end{tabular}

Figura 3.

Acceso de usuarios al Sistema Nacional de Bibliotecas

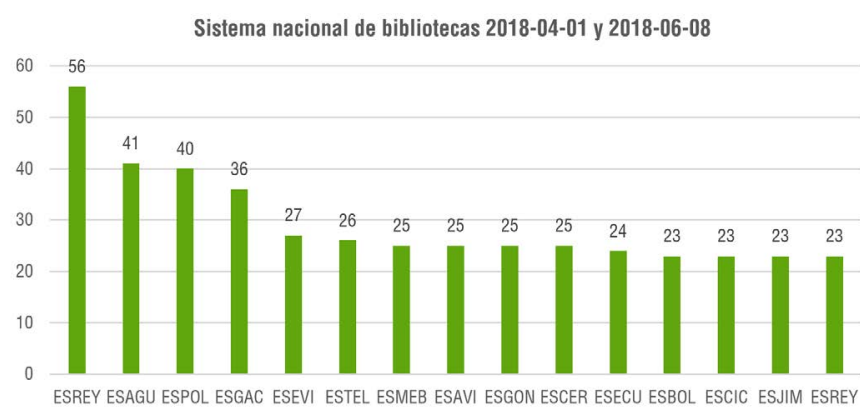

Otro de los insumos utilizados en la fase de diagnóstico fueron los resultados obtenidos en la matriz del Ministerio de Defensa, donde se miden las capacidades tecnológicas de la DINAE, evaluadas por el director TIC. En este instrumento se evidencian las capacidades que han sido priorizadas desde la Dirección, y que deben ser aprovechadas y potenciadas en el marco de la elaboración del ecosistema digital (figura 4).

Este formulario está enfocado en conseguir la información asociada, únicamente, a cada una de las capacidades priorizadas que encuentra en el recuadro superior. En otras palabras, no indaga acerca de la totalidad de la unidad, sino respecto al desarrollo de las capacidades mencionadas. De este modo, señala factores relacionados con la infraestructura, resultado que corrobora la percepción y alta valoración de los usuarios acerca de este componente de las TIC. Empero, no se observan en las capacidades priorizadas en los procesos académicos de la DINAE.

Figura 4

Matriz de seguimiento y evaluación: herramientas TIC en la educación

\section{Información de contacto de la unidad}

1. Fuerza

PNC

2. Nombre de la escuela

Dirección Nacional de Escuelas

3. Cargo de la persona/uniformado que diligencia el formulario Jefe Grupo Telemática DINAE

4. Ubicación de la unidad (Departamento)

$$
\text { Cundinamarca }
$$

5. Ubicación de la unidad (Municipio/ciudad)

BOGOTÁ D.C.

\section{Capacidades Priorizadas}

\section{Capacidad de soporte}

Arquitectura empresarial de TIC

Canales dedicados de datos

Red Satelital

Data Center (Centro de cómputo)

Sistemas de energía convencionales, alternativos y sostenibles

Sistemas de videovigilancia, reconocimiento y circuitos cerrados de televisión

Sistemas de información (Sectoriales e institucionales)

Soporte de redes de telecomunicaciones y datos

Soporte de hardware y software

Soporte a Ciberseguridad PNC
Numeración Avance

TIC 4

TIC 11

TIC 15

TIC 20

TIC 21

TIC 26

TIC 29

TIC 46

TIC 47

TIC 52
$100 \%$ $100 \%$ $81 \%$ $82 \%$ $81 \%$ $81 \%$ $81 \%$ $81 \%$ $75 \%$ $81 \%$ 
Con los instrumentos aplicados y analizados en la fase de diagnóstico se lograron identificar las principales necesidades de los usuarios sobre servicios, infraestructura, información y ofertas de formación de las diferentes plataformas existentes en la DINAE. Esto permitió definir la manera de articularlas y optimizarlas en un ecosistema digital, tal como se presenta a continuación.

Fase II. Diseño basado en el Proyecto Educativo Institucional de la Policía

El ecosistema digital se planteó con el propósito de apoyar los procesos educativos de la DINAE de la Policía Nacional, y en él se articulan, en un único espacio digital, los diferentes servicios de las escuelas de la Policía. En este apartado se exponen y describen los diferentes elementos que conforman el diseño de la propuesta.

\section{Descripción del diseño del ecosistema digital}

El diseño del ecosistema digital, en su componente de servicios académicos digitales, comprende las posibilidades y limitaciones de los recursos ofertados a docentes y estudiantes para representar, procesar, transmitir y compartir información. Este diseño resultó ser un espacio digital adecuado para 28 escuelas, dado el proceso de convergencia y usabilidad oportuna y efectiva que arrojaron los resultados de uso de la plataforma a favor de las labores realizadas en el sitio web de la institución de la Policía Nacional (figura 5).

Figura 5

Página web de la Dirección Nacional de Escuela

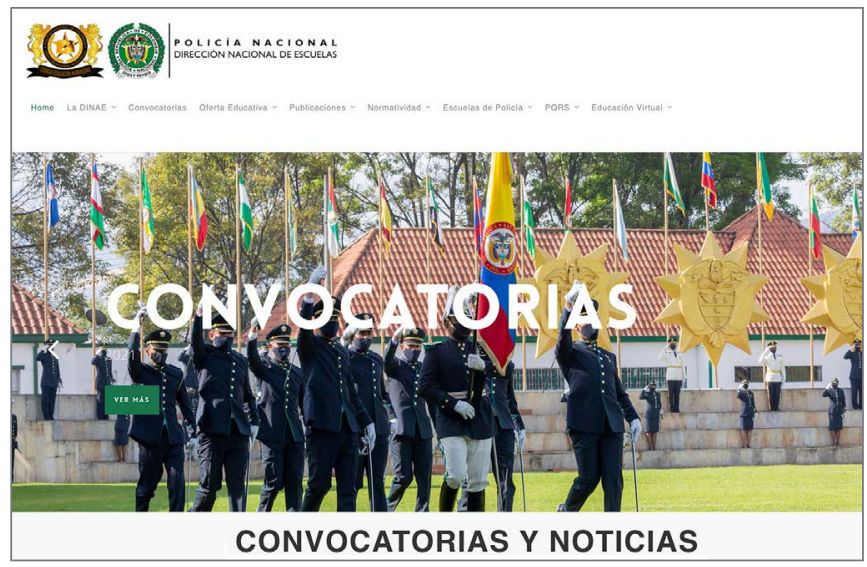

Nota: http://www.policia.edu.co

Este espacio en el sitio web fue otorgado después de hacer evidente la necesidad del ecosistema digital. El diagnóstico mostró que tener los servicios alojados en distintos sitios no resulta efectivo, pues se les da poco uso y no se apunta a su integralidad. En otras palabras, se pudo evidenciar la importancia de generar un entorno donde se alojen varios servicios que implican el manejo de herramientas, recursos, tiempo y dominio de la información sobre el uso de las TIC.

Teniendo en cuenta la organización y estructura de la página web, en la cual se incrustó la aplicación de servicios del ecosistema digital, se diseñó un escenario mediante vínculos y botones que permiten guardar la seriedad y la línea gráfica establecidas por la institución. Por esta razón, el ecosistema se convirtió en un espacio de fácil acceso para múltiples plataformas (computadores, tabletas, celulares) (figura 6).

Figura 6

Botón de oferta educativa, ecosistema digital en la página de la Policía Nacional

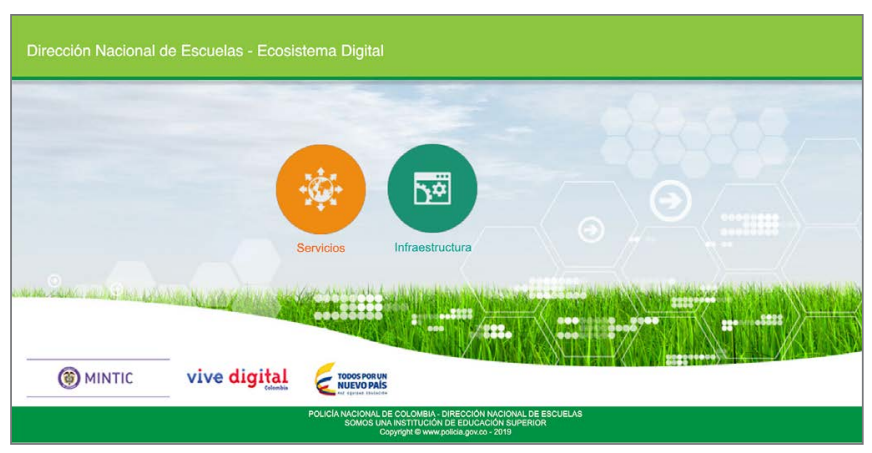

Nota: http://www.policia.edu.co

Esta dinámica tiene lugar en un ecosistema móvil (Aguado \& Navarro, 2013), hegemonizado por dispositivos portátiles que se han convertido en tecnologías relacionales, donde el contenido no es un paquete cerrado que se entrega a los consumidores a través de una tecnología de distribución; por el contrario, es un contenido que circula y se inserta en las conversaciones que mantienen los usuarios conectados ubicuamente a la red (Jenkins, Ford \& Green, 2013).

Por consiguiente, el diseño del ecosistema digital se genera desde el ambiente natural de ciertos servicios que se prestan en la DINAE de la Policía Nacional, transformado por las tecnologías digitales. Para esto, se creó un ambiente materializado en íconos, para la organización de contenido (ver figura 7). Este ambiente está basado en una estructura dinámica y en la participación colaborativa, la validación y el soporte tecnológico relacional donde se encuentra: 
1. Sistema Nacional de Bibliotecas y bases de datos especializadas.

2. Red Nacional Académica de Tecnología Avanzada (RENATA).

3. Acceso a las herramientas $G$ Suite.

4. Acceso a la Revista Logos Ciencia \& Tecnología.

5. Asesoría para la mediación pedagógica y tecnológica de eventos académicos.

6. Plataforma educativa virtual MOODLE.

7. Portal de Servicio Interno (PSI).

8. Repositorios de recursos educativos abiertos.

Figura 7

Convergencia de servicios del ecosistema digital en la página de la Policía Nacional

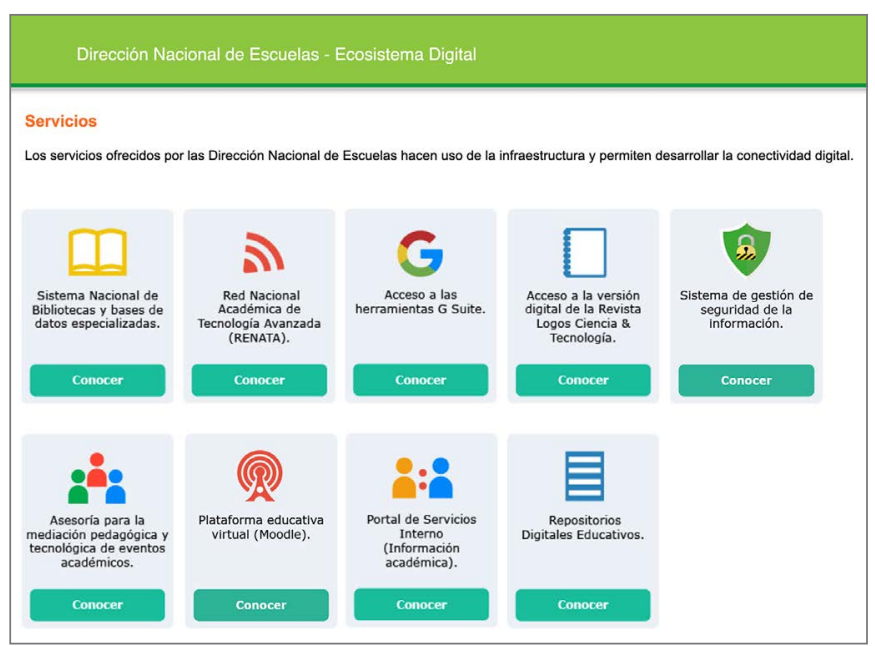

Nota: http://www.policia.edu.co

\section{Descripción del diseño gráfico del ecosistema}

El diseño está basado en la tendencia Flat UI (Interaction Design Foundation, 2019), donde el contenido de cualquier sitio web se escala, sin problemas, dependiendo del tamaño de la pantalla del dispositivo. Con el uso de formas simples y texturas mínimas, el diseño plano garantiza que las interfaces funcionen bien y se carguen rápidamente; este aspecto es importante, porque los dispositivos móviles tienen velocidades de internet más lentas.

Al reducir la cantidad de ruido visual (en forma de texturas y sombras), el diseño plano proporciona a los usuarios una experiencia de usuario optimizada y eficaz. Esto genera contenidos más rápidos, de menor peso y con características como:
- Ausencia de texturas, sombras, gradientes, biseles o cualquier otro elemento que provoque sensación de profundidad.

- Diseño minimalista, en el que los elementos "respiran" y no se amontonan uno sobre otro.

- Uso de formas geométricas simples (cuadrados, rectángulos, círculos, etc.) para estructurar la información o diseñar elementos de la interfaz (botones, iconos, etc.).

- La tipografía adopta un papel protagonista en el diseño plano, con preferencia por familias sin serifas (de palo seco), en diferentes tamaños y variantes.

- La paleta de colores se amplía en su formato más puro: colores primarios y secundarios.

Este tipo de diseño permite mejorar los tiempos de carga de la web y reescalar fácilmente los elementos en pantalla, al ser ligero y rápido; el lenguaje de programación (HTML5) y las herramientas empleadas logran una máxima compatibilidad con el diseño adaptativo (Responsive Web Design). Es un diseño que ofrece una mayor cobertura, al incluir los dispositivos móviles.

\section{Conclusiones}

En el marco de los resultados obtenidos en el diagnóstico, se constató la necesidad de poner en marcha una solución integradora de los diferentes servicios formativos que ofrece la DINAE de la Policía Nacional de Colombia. Antes de materializar esta propuesta, los procesos relacionados con este componente se encontraban desarticulados entre sí, siendo poco conocidos o consultados por los usuarios.

El ecosistema digital se ha utilizado para comparar los sistemas empresariales con los sistemas naturales y analizar cómo se interrelacionan las redes, el internet y las plataformas. En virtud de ello, se diseñó para la Policía Nacional una estrategia para articular la prestación del servicio y generar buenas prácticas de información, participación, colaboración, búsqueda y construcción de canales alternativos y eficaces.

Como una respuesta al plan de acción de la vigencia 2017 y 2018 de la DINAE, se estipuló la necesidad de implementar y socializar un insumo real para la comunidad académica. Este requerimiento fue avalado y registrado 
en la herramienta de medición de órdenes (Suite Visión Empresarial) y en las tareas del grupo de despliegue telemático, relacionadas con los ejercicios de desarrollo de ecosistemas digitales 4.1 y 4.2 .

Lo anterior fue una oportunidad para poner en tensión las prácticas tecno-pedagógicas asumidas en la institución, con el propósito de buscar estrategias que permitan responder de forma efectiva a los intereses pedagógicos de las generaciones actuales (Coll, 2013). Por esta razón, con la construcción del ecosistema digital se espera generar valor sobre los servicios académicos de la Policía, orientados a mejorar las interacciones de los miembros desde la aplicación en convergencia de ocho componentes alojados en el portal educativo institucional.

El diseño y puesta en marcha de este ecosistema digital permitió mejorar la gestión tecnológica de la institución y los procesos operacionales, revalorando la administración y percepción de servicio al cliente a nivel interno. Además, se proyecta que la institución logre mejorar los procesos de visibilidad, comunicación y posicionamiento en la ciudadanía. Si la Policía Nacional de Colombia expande su ecosistema, tendrá mayor control y monitoreo sobre las herramientas, aplicaciones y el medio con el que cuenta, logrando mayor cobertura en el país, mientras responde a las exigencias más vanguardistas del desarrollo (Policia Nacional de Colombia, 2015).

Sin duda, este proyecto y su materialización representan un paso para fortalecer las fuentes de información, la accesibilidad y la visibilidad de las capacidades institucionales, de cara a las nuevas tendencias y desafíos de la sociedad actual. Con el ecosistema digital se logran enriquecer las experiencias de aprendizaje de los usuarios académicos de la DINAE, y se brindan herramientas, actividades interactivas, simulaciones y juegos, para la formación de policías comprometidos con su misión constitucional.

\section{Referencias}

Aguado, J., \& Navarro, H. (2013). Comunicación móvil, ecosistema digital e industrias culturales. En J. Aguado, C. Feijoó, \& I. Martínez (coord.) La comunicación móvil. Hacia un nuevo sistema digital pp. 57-78. Gedisa.

Barrios, L., \& Chaves, M. (2016). El proyecto de aula como estrategia didáctica en el marco de la enseñanza para la comprensión. Avances en Educación y Humanidades, 1(1), 39-54.
Cáceres, M., Brändle, G., \& Ruiz, J. (2016). Sociabilidad virtual: la interacción social en el ecosistema digital. Historia y Comunicación Social, 22(1), 233-247. https://doi.org/10.5209/HICS.55910

Carrera, P., Limón, N., Herrera, E., \& De Baranda, C. (2013). Transmedialidad y ecosistema digital. Historia y Comunicación Social, 18, 535-545.

Coll, C. (2013). El currículo escolar en el marco de la nueva ecología del aprendizaje. Aula de Innovación Educativa, (219), 31-36.

Díaz, D. (2016). Diseño de un proyecto integrador que incorpora TIC desde el enfoque pedagógico sociocrítico con los docentes de ciclo cuatro de la Institución Educativa Distrital Eduardo Umaña Mendoza [Tesis de maestría, Universidad de La Sabana]. Recuperado de: https://intellectum.unisabana.edu.co/ handle/10818/29979? show=full

Fundación Telefónica. (2016). Las reglas del juego en el ecosistema digital. https://www.fundaciontelefonica. com/arte_cultura/publicaciones-listado/pagina-itempublicaciones/itempubli/501/

Hernández, R., Fernández, C.,\& Baptista, M. (2015). Metodología de la investigación. McGraw Hill.

Interaction Design Foundation. (2019). What is Flat Design? https://www.interaction-design.org/literature/topics/flat-design

Islas, C. (2017). La implicación de las TIC en la educación: alcances, limitaciones y prospectiva. Revista Iberoamericana para la Investigación y el Desarrollo Educativo, 8(15), 861-876. https://doi.org/10.23913/ ride.v8i15.324

Islas, C. (2019). Los ecosistemas de aprendizaje y estudiantes universitarios: una propuesta de abordaje sistémico. Revista de Psicología y Ciencias del Comportamiento, 10(2), 172-186.

ITU (2017). Estudios de caso: el ecosistema digital y la masificación de las Tecnologías de la Información y las Comunicaciones (TIC) en Panamá. https://www.itu. int/dms_pub/itu-d/opb/pref/D-PREF-EF.CS_PANAMA2017-PDF-S.pdf

Jenkins, H., Ford, S., \& Green, J. (2013). Spreadable media. Creating value and meaning in a networked culture. NYU Press. https://www.jstor.org/stable/j.ctt9qfk6w

Jiménez, I. (2015). Pedagogía de la creatividad viable: un camino para potencializar el pensamiento crítico. Opción, 31(2), 632-653. 
Jiménez, I. (2018). Didácticas innovadoras mediadas por TIC: retos a partir de la ecología del aprendizaje [Proyecto de investigación, Universidad de La Sabana].

Jiménez, I. (2020). El triángulo lógico: una ecuación didáctica emergente para aprender metodología de la investigación. Ed. Universidad de La Sabana.

López, G. (2005). El ecosistema digital: modelos de comunicación, nuevos medios y público en internet. Ed. Universitat de València.

Magro, C., Salvatella, J., Álvarez, M., Herrero, O., Paredes, A., \& Vélez, G. (2014). Cultura digital y transformación de las organizaciones. Roca Salvatella.

MinTIC (2018a). Plan TIC: 2018-2020. Ministerio de las Tecnologías de la Información y la Comunicación.

MinTIC (2018b). Subdirección de Cultura Digital. https:// mincultura.gov.co/areas/comunicaciones/cultura-digital/Paginas/default.aspx

MinTIC (2019). Ecosistema Digital. https://mintic.gov.co/ portal/vivedigital/612/w3-propertyvalue-634.html

Morales, S. (2018). Nuevos ecosistemas digitales en el nivel universitario : el caso MOOC : una aproximación al aprendizaje en los estudios de nivel superior [Trabajo final integrador, Universidad Nacional de Quilmes]. https://ridaa.unq.edu.ar/handle/20.500.11807/911

Motz, R., \& Rodés, V. (2013). Pensando los Ecosistemas de Aprendizaje desde los Entornos Virtuales de Aprendizaje. Conferencias LACLO.
Ortiz, M., Muñoz, J., Canul, J., \& Broisin, J. (2017). Análisis de uso de un ecosistema digital como apoyo a niños con problemas de aprendizaje en lectura y matemáticas básicas. Campus Virtuales, 6(2), 91-105.

Ospina, Y., \& Galvis, J. (2017). Un diseño novedoso de un ecosistema digital para educación virtual. Tekhnê, 14(1), 55-60.

Pineda, E., \& Orozco, P. (2017). Ecosistemas de aprendizaje con gestión de TIC. Una estrategia de formación desde la pedagogía praxeológica. Revista Docencia Universitaria, 17, 71-95.

Policía Nacional de Colombia (2015). Plan estratégico institucional: comunidades seguras y en paz. https:// www.policia.gov.co/sites/default/files/descargables/ plan-estrategico-institucional-2015-2018.pdf

Policía Nacional de Colombia (2019). Página de la Policía Nacional de Colombia. https://www.policia.gov.co/direcciones/educacion-policial

Prensky, M. (2001). Digital Natives, Digital Immigrants Part 1. On the Horizon, 9(5), 1-6. https://doi. org/10.1108/10748120110424816

Ramírez, M., \& Chacón, C. (2018). Inmersión en los ecosistemas digitales, una forma de mejora de la educación en las áreas rurales en Colombia. Realidades y Visiones Sobre Latinoamérica, 8, 60-67.

Rey, G. (2013). Del ecosistema mediático al ecosistema digital en Colombia. Telos. Revista de Pensamiento Sobre Comunicación, Tecnología y Sociedad, 97, 16-25. 